\title{
Recherche de facteurs limitant la nutrition minérale de l'orge en milieu salé
}

\author{
A Soltani 1, M Haiji 1, C Grignon 2 \\ 1 Institut National de Recherche Scientifique et Technique (INRST), BP 95, Hammam Lif, 2050, Tunisie; \\ 2 ENSA-INRA, Biochimie et Physiologie Végétales, CNRS URA 573, 34060 Montpellier Cedex 1, France
}

(Reçu le 13 février 1990; accepté le 10 octobre 1990)

\begin{abstract}
Résumé - Le but de cette étude est de déterminer chez de jeunes plantes d'orge (Hordeum vulgare L), âgées de 12 jours, si l'application d'une forte dose de $\mathrm{NaCl}(200 \mathrm{mM})$, correspondant au seuil de tolérance de cette espèce, rend les éléments $\mathrm{N}, \mathrm{K}$ ou Ca limitants pour la croissance. La technique dite d'alimentation mixte permet d'obtenir à la fois des tissus riches en $\mathrm{Na}^{+}$et $\mathrm{Cl}^{-}$, et une alimentation correcte en nutriments essentiels. Cette méthode permet de dissocier aisément les deux niveaux d'action du sel, celui du milieu, et celui accumulé dans les tissus. Les fortes accumulations de $\mathrm{Na}^{+}$et $\mathrm{Cl}^{-}$dans les tissus s'avèrent sans rapport direct avec la diminution de la croissance et de l'alimentation en $\mathrm{NO}_{3}^{-}, \mathrm{K}^{+}$et $\mathrm{Ca}^{2+}$. La présence de $\mathrm{NaCl}$ dans le milieu de culture réduit fortement l'absorption et le transport des éléments majeurs : la fourniture de $\mathrm{NO}_{3}-$ et de $\mathrm{Ca}^{2+}$ ne satisfait plus les besoins de la croissance; cependant, celle de $\mathrm{K}^{+}$, quoique très réduite, paraît suffisante.
\end{abstract}

orge / NaCl / nitrate / potassium / calcium

Summary - Factors which limit the mineral nutrition of barley in the presence of NaCl. The purpose of this work was to determine whether the growth of 12-day-old barley seedlings was limited by $N, K$, or Ca when the medium contained high $\mathrm{NaCl}$ concentration (200 $\mathrm{mM}$, the highest concentration tolerated by barley). Plants with split root systems were used to obtain high $\mathrm{Na}^{+}$and $\mathrm{Cr}^{-}$contents in tissues, and simultaneous normal provision with other ions (tables I and II). This method allows a distinction to be made between the effects of $\mathrm{NaCl}$ in the medium and those of $\mathrm{Na}^{+}$and $\mathrm{Cr}$ in the tissues. The high accumulation levels of $\mathrm{Na}^{+}$and $\mathrm{Cr}^{-}$in the tissues did not seem to be responsible for the reduction of growth (table III), or for the decrease in uptake of $\mathrm{NO}_{3}^{-}$(table $\mathrm{IV}$ ), $\mathrm{K}^{+}$(fig 4), and Ca2+ (fig 6). The uptake and transport of the main elements were strongly reduced when $\mathrm{NaCl}$ was present in the medium of the absorbing roots. $N$ (provided as nitrate, fig 1), and $\mathrm{Ca}^{2+}$ (fig 6) were growth-limiting, but this was not the case for $\mathrm{K}^{+}$(figs 4 and 5).

barley / $\mathrm{NaCl} /$ nitrate / potassium / calcium

\section{INTRODUCTION}

L'orge est réputée tolérante au sel. En milieu hydroponique, sa croissance n'est affectée que par des concentrations de $\mathrm{NaCl}$ au moins égales à environ $200 \mathrm{mM}\left(12 \mathrm{~g}^{\cdot 1}{ }^{-1}\right)$. La réduction de croissance s'accompagne alors d'une augmentation de l'accumulation de $\mathrm{Na}^{+}$et de $\mathrm{Cl}$ - dans les tissus, et d'une diminution de $\mathrm{K}^{+}$et $\mathrm{Ca}^{2+}$ (Greenway, 1962a, b et 1963; Munns et al, 1982). L'effet de $\mathrm{NaCl}$ dépend des concentrations de $\mathrm{K}^{+}$et $\mathrm{Ca}^{2+}$ dans le milieu de culture (Heyder et Greenway, 1965; Elzam, 1971). Le rapport $\mathrm{Na}+/ \mathrm{Ca}^{2}+$ semble également être un déterminant important du comportement des plantes (Cramer et al, 1986). Au niveau des racines, $\mathrm{Na}^{+}$déplace $\mathrm{Ca}^{2}+$ des parois cellulaires (Soll et Bottger, 1982). Dans les parois isolées de racines d'orge, $\mathrm{Na}^{+}$et $\mathrm{Ca}^{2}+$ sont en compéti- tion pour les mêmes sites d'adsorption, tandis que $\mathrm{K}^{+}$est fixé sur d'autres sites (Stassart et al, 1981). Il en résulte que le rapport $\mathrm{K}+/ \mathrm{Na}^{+}$à la surface des cellules dépend de la compétition $\mathrm{Na}^{+} / \mathrm{Ca}^{2+}$, ce qui se répercute directement sur l'absorption endocellulaire des deux monovalents. Un autre exemple de compétition $\mathrm{Na}^{+} /$ $\mathrm{Ca}^{2+}$ pour les sites apoplasmiques est fourni par les feuilles de Citrus, où le déplacement de $\mathrm{Ca}^{2+}$ par $\mathrm{Na}^{+}$de ses sites d'adsorption entraîne des nécroses du limbe (Zid et Grignon, 1985). L'utilisation de sondes fluorescentes a permis de montrer que $\mathrm{Na}^{+}$déplace également $\mathrm{Ca}^{2+}$ de la membrane plasmique des poils absorbants (Cramer et al, 1985), ainsi que les compartiments de stockage endocellulaires des cellules corticales de racines (Lynch et Läuchli, 1988). II s'ensuit une altération de la sélectivité $\mathrm{K}+/ \mathrm{Na}^{+}$de l'absorption, et une perte de $\mathrm{K}^{+}$des tissus (La Haye 
et Epstein, 1969; Kent et Läuchli, 1985; Haiji et Grignon, 1985). Tous ces résultats suggèrent que $\mathrm{K}^{+}$et $\mathrm{Ca}^{2+}$ pourraient devenir des facteurs limitants pour la croissance quand le milieu est enrichi en $\mathrm{NaCl}$.

De même, l'absorption et l'assimilation de $\mathrm{NO}_{3}^{-}$sont diminuées chez l'orge cultivée en présence de $\mathrm{NaCl}$ (Helal et Mengel, 1979; Lucque et Bingham, 1981; Aslam et al, 1984). On sait que $\mathrm{Cl}$ inhibe l'absorption de $\mathrm{NO}_{3}^{-}$(DeaneDrummond et Glass, 1982). Enfin, chez certaines plantes, un apport supplémentaire de $\mathrm{NO}_{3}^{-}$dans le milieu stimule davantage la croissance des plantes traitées par $\mathrm{NaCl}$ que celle des plantes non traitées (Langdale et Thomas, 1971). Pourtant, la limitation de la croissance par la faible disponibilité de $\mathrm{NO}_{3}^{-}$en milieu salé est loin d'être établie, étant donné la variété de données contradictoires sur ce point (Munns et Termaat, 1986).

Effectivement, la restriction par $\mathrm{NaCl}$ de l'alimentation des plantes en $\mathrm{K}, \mathrm{Ca}$, et $\mathrm{N}$ (qui ne fait aucun doute), n'implique pas obligatoirement que ces éléments deviennent limitants pour la croissance. Pour tenter de préciser ce point, nous avons adopté la méthode dite d'alimentation mixte (Hajji, 1980) ou split-root system (Lambers et al, 1982) : le système racinaire de chaque plante est divisé en deux parties, chacune plongeant dans un milieu distinct. Nous avons ainsi cultivé des plants d'orge dans des conditions telles que $\mathrm{NaCl}$ ne puisse affecter l'absorption que d'un seul élément ( $K, \mathrm{Ca}$ ou $N)$, la fourniture des autres éléments restant assurée par les racines dans un milieu sans $\mathrm{NaCl}$.

\section{MATÉRIEL ET MÉTHODES}

Les expériences sont faites sur l'orge Martin (Hordeum vulgare $\mathrm{L}$ ), variété fréquemment cultivée en Tunisie. Les cultures sont conduites sur milieu liquide dans les conditions décrites précédemment (Soltani et al, 1989). Le milieu de base $T$ est exclusivement nitrique (Soltani et al, 1989, milieu $\mathrm{NO}_{3}^{-}$, type 1). Outre les oligoéléments et $\mathrm{Fe}$, il contient $(\mathrm{mM}): \mathrm{K}^{+}(2,2)$; $\mathrm{Ca}^{2+}$ $(2,0) ; \mathrm{Mg}^{2+}(1,5) ; \mathrm{NO}_{3}^{-}(4,0) ; \mathrm{H}_{2} \mathrm{PO}_{4}^{-}$plus $\mathrm{HPO}_{4}^{2-}(2,2)$; $\mathrm{SO}_{4}^{2-}(1,5)$. À l'apparition de la seconde feuille (12 j après germination), les plantes sont soumises aux différents traitements d'alimentation mixte. Cette technique consiste à cultiver des plantes avec leurs systèmes racinaires divisés en deux parties équivalentes. L'une est placée sur $200 \mathrm{ml}$ de milieu complet avec éventuellement $\mathrm{NaCl}(200 \mathrm{mM})$; l'autre partie est sur $200 \mathrm{ml}$ de milieu dépourvu de l'un des éléments étudiés ( $\mathrm{N}, \mathrm{K}$ ou $\mathrm{Ca}$ ).
Pour l'étude de la nutrition azotée, les cultures sont faites pendant 12 j sur eau distillée afin d'épuiser les réserves de la graine et de disposer de plantules pauvres en azote; les plantes sont ensuite soumises au traitement d'alimentation mixte (tableau I). Les milieux sans azote (TON et T12 ON) sont obtenus par remplacement de $\mathrm{Ca}\left(\mathrm{NO}_{3}\right)_{2}$ par $2 \mathrm{mM} \mathrm{CaSO}_{4}$, dans le milieu de base $\mathrm{T}$.

Pour l'étude des effets de $\mathrm{NaCl}$ sur l'alimentation en $\mathrm{K}$ ou en $\mathrm{Ca}$, les plantes sont cultivées pendant $12 \mathrm{j}$ sur milieu de base $T$ puis soumises aux traitements d'alimentation mixte (tableau II). Les milieux sans potassium (TOK) sont obtenus par remplacement de $\mathrm{KH}_{2} \mathrm{PO}_{4}$ et de $\mathrm{K}_{2} \mathrm{HPO}_{4}$ par la même concentration équivalentaire en $\mathrm{Ca}\left(\mathrm{H}_{2} \mathrm{PO}_{4}\right)_{2}$ et $\mathrm{Ca}\left(\mathrm{HPO}_{4}\right)$. Les milieux sans calcium (TOCa et $\mathrm{T} 12 \mathrm{OCa}$ ) sont obtenus par remplacement de $\mathrm{Ca}\left(\mathrm{NO}_{3}\right)_{2} 2 \mathrm{mM}$ par $\mathrm{KNO}_{3}, 4$ $\mathrm{mM}$. L'effet de la déficience en $\mathrm{K}$ a été également étudié par un série d'expériences dans lesquelles on a procédé à un apport supplémentaire de $\mathrm{K}\left(\left.8 \mathrm{mmol} \cdot\right|^{-1}\right.$ de $\mathrm{KCl}$ ou $4 \mathrm{mmol}^{-1}$ de $\mathrm{K}_{2} \mathrm{SO}_{4}$ ) aux milieux de culture qui contiennent $\mathrm{NaCl}$. Ces expériences ont été faites sous un éclairement deux fois plus faible que les autres, ce qui explique les différences de croissance des plantes témoins entre les figures 4 et 6 d'une part, et 5 d'autre part.

Les prélèvements sont effectués au début des traitements (12 j), et à la fin de l'expérience (24j). Les paramètres étudiés sont la croissance, l'absorption, le transport et l'accumulation des cations $\left(\mathrm{K}+, \mathrm{Na}^{+}, \mathrm{Ca}^{2+}\right.$, $\left.\mathrm{Mg}^{2+}\right)$, des anions $\left(\mathrm{NO}_{3}^{-}, \mathrm{Cl}-, \mathrm{SO}_{4}^{2-}, \mathrm{Pi}\right)$ et des différentes fractions azotées. Les techniques analytiques ont été décrites précédemment (Soltani et al, 1989). Pour chaque traitement les mesures sont faites sur 5 plantes (feuilles et racines séparées).

\section{RÉSULTATS}

\section{Accumulation de $\mathrm{Na}^{+}$et $\mathrm{Cr}$}

Le tableau III montre les accumulations de $\mathrm{Na}^{+}$ et $\mathrm{Cl}^{-}$dans les feuilles, mesurées à la récolte finale pour les traitements d'alimentation mixte. II indique aussi les masses finales des feuilles (MS) tirées des figures 1 et 6 , exprimées en \% des témoins sans $\mathrm{NaCl}$. On voit que les fortes teneurs en $\mathrm{Na}^{+}$et $\mathrm{Cl}^{-}$obtenues dans les traitements mixtes ne sont pas corrélées avec une baisse de la croissance. Ceci indique que les diminutions de croissance en présence de $\mathrm{NaCl}$ sont attribuables aux effets du sel dans le milieu plutôt qu'à un effet toxique propre des ions $\mathrm{Na}^{+}$ et $\mathrm{Cl}^{-}$accumulés. L'hypothèse éprouvée dans ce qui suit est que $\mathrm{NaCl}$ dans le milieu restreint l'absorption des nutriments par la racine. 
Tableau I. Protocoles expérimentaux pour l'étude de l'effet de $\mathrm{NaCl}$ sur l'utilisation de $\mathrm{NO}_{3}^{F}$ La dénomination T1/T2 indique les milieux sur lesquels sont placées respectivement les deux moitiés (1) et (2) du système racinaire de chaque plante. T : milieu de base complet; T ON : milieu de base sans azote; T12 : milieu de base complet avec $\mathrm{NaCl}$ (200 $\mathrm{mM}) ; \mathrm{T} 12$ oN : milieu T12 sans azote.

\begin{tabular}{|c|c|c|c|c|}
\hline \multirow{2}{*}{$\begin{array}{l}\text { Traitement } \\
\quad T 1 / T 2\end{array}$} & \multirow{2}{*}{$N^{\circ}$} & \multicolumn{2}{|c|}{$\begin{array}{l}\text { Caractéristiques des } \\
\text { milieux des racines }\end{array}$} & \multirow{2}{*}{ Objectif } \\
\hline & & Racine 1 & Racine 2 & \\
\hline $\begin{array}{l}\mathrm{T} / \mathrm{T} \\
\mathrm{T} / \mathrm{T} \text { ON }\end{array}$ & $\begin{array}{l}(1) \\
(2)\end{array}$ & $\begin{array}{l}\mathrm{NO}_{3} \\
\mathrm{NO}_{3}\end{array}$ & $\begin{array}{l}\mathrm{NO}_{3} \\
\mathrm{O} \mathrm{NO}_{3}\end{array}$ & $\begin{array}{l}\text { Témoin sans sel } \\
\text { Absence de } \mathrm{N} \text { sur une partie du système racinaire }\end{array}$ \\
\hline $\mathrm{T} / \mathrm{T} 12 \mathrm{ON}$ & (3) & $\mathrm{NO}_{3}$ & $0 \mathrm{NO}_{3}+\mathrm{NaCl}$ & $\begin{array}{l}\text { Absence de } \mathrm{N} \text { et présence de } \mathrm{NaCl} \\
\text { sur une même partie du système racinaire }\end{array}$ \\
\hline T12/T ON & (4) & $\mathrm{ONO}_{3}$ & $\mathrm{NO}_{3}+\mathrm{NaCl}$ & $\begin{array}{l}\text { Double contrainte : } \\
\text { absence de } \mathrm{N} \text { sur une partie du système racinaire, } \\
\text { et présence de } \mathrm{NaCl} \text { sur l'autre. }\end{array}$ \\
\hline $\mathrm{T} 12 / \mathrm{T} 12 \mathrm{ON}$ & (5) & $\mathrm{NO}_{3}+\mathrm{NaCl}$ & $0 \mathrm{NO}_{3}+\mathrm{NaCl}$ & Comme ci-dessus, mais contrainte plus sévère \\
\hline $\mathrm{T} 12 \mathrm{~T} 12$ & (6) & $\mathrm{NO}_{3}+\mathrm{NaCl}$ & $\mathrm{NO}_{3}+\mathrm{NaCl}$ & Témoin avec $\mathrm{NaCl}$ \\
\hline
\end{tabular}

Tableau II. Protocoles expérimentaux pour l'étude de l'effet de l'absence de $\mathrm{Ca}^{2+}$ ou de $\mathrm{K}+$ dans le milieu de culture. La dénomination $\mathrm{T1} / \mathrm{T} 2$ indique les milieux sur lesquels sont placées respectivement les deux moitiés (1) et (2) du système racinaire de chaque plante. $\mathrm{T}$ : milieu de base complet; $\mathrm{T} 12$ : milieu de base complet avec $\mathrm{NaCl}(200 \mathrm{mM})$; T OK : milieu de base sans K; T OCa : milieu de base sans $\mathrm{Ca}$; T12 0Ca : milieu T12 sans Ca.

\begin{tabular}{|c|c|c|c|}
\hline \multirow{2}{*}{$\begin{array}{c}T \text { traitement } \\
T 1 / T 2\end{array}$} & \multicolumn{2}{|c|}{$\begin{array}{l}\text { Caractéristiques des } \\
\text { milieux des racines }\end{array}$} & \multirow{2}{*}{ Objectif } \\
\hline & Racine 1 & Racine 2 & \\
\hline $\begin{array}{l}\mathrm{T} / \mathrm{T} \\
\mathrm{T} 12 / \mathrm{T} 12 \\
\mathrm{~T} / \mathrm{T} 12 \\
\mathrm{~T} / \mathrm{T} \text { OK } \\
\mathrm{T} 0 \mathrm{~K} / \mathrm{T} 12 \\
\mathrm{~T} / \mathrm{T} \text { OCa } \\
\mathrm{T} 12 / \mathrm{T} \text { OCa } \\
\mathrm{T} / \mathrm{T} 12 \text { OCa } \\
\mathrm{T} 12 / \mathrm{T} 12 \mathrm{OCa}\end{array}$ & $\begin{array}{l}\text { Complet sans } \mathrm{NaCl} \\
\text { Complet }+\mathrm{NaCl} \\
\text { Complet sans } \mathrm{NaCl} \\
\text { Complet sans } \mathrm{NaCl} \\
\text { Complet }+\mathrm{NaCl} \\
\text { Complet sans } \mathrm{NaCl} \\
\text { Complet }+\mathrm{NaCl} \\
\text { Complet sans } \mathrm{NaCl} \\
\text { Complet }+\mathrm{NaCl}\end{array}$ & $\begin{array}{l}\text { Complet sans } \mathrm{NaCl} \\
\text { Complet }+\mathrm{NaCl} \\
\text { Complet }+\mathrm{NaCl} \\
\text { Complet sans } \mathrm{K} \\
\text { Complet sans } \mathrm{K} \\
\text { Complet sans } \mathrm{Ca} \\
\text { Complet sans } \mathrm{Ca} \\
+\mathrm{NaCl} \text { sans } \mathrm{Ca} \\
+\mathrm{NaCl} \text { sans } \mathrm{Ca}\end{array}$ & $\begin{array}{l}\text { Témoin nutrition normale } \\
\text { Témoin avec } \mathrm{NaCl} \\
\text { Effet de } \mathrm{NaCl} \text { accumulé } \\
\text { Témoin du suivant } \\
\text { Effet de } \mathrm{NaCl} \text { sur l'absorption de } \mathrm{K} \\
\text { Témoin du suivant } \\
\text { Effet de } \mathrm{NaCl} \text { sur l'absorption de } \mathrm{Ca} \\
\text { Rôle protecteur de } \mathrm{Ca} \\
\text { Rôle protecteur de } \mathrm{Ca}\end{array}$ \\
\hline
\end{tabular}

\section{Alimentation en azote}

\section{Croissance}

La figure 1 représente les masses des feuilles et des racines des plantes soumises à deux catégories de traitements : ceux qui autorisent l'absorption de $\mathrm{NO}_{3}^{-}$en l'absence de $\mathrm{NaCl}$ (T/T, T/TON, $\mathrm{T} / \mathrm{T} 12 \mathrm{ON}$ ) et ceux qui obligent la plante à prélever $\mathrm{NO}_{3}^{-}$sur milieu contenant $\mathrm{NaCl}(\mathrm{T} 12 / \mathrm{T} 12$, $\mathrm{T} 12 / \mathrm{T}$ ON et T12/T12 ON). La suppression de la fourniture de $\mathrm{NO}_{3}^{-}$, en absence comme en pré- sence de $\mathrm{NaCl}$, à une moitié du système racinaire (R2 milieux T ON ou T12 ON) entraîne une croissance disproportionnée de l'autre moitié (R1 sur $\mathrm{T}$, fig 1), de telle sorte que la masse totale des racines s'en trouve augmentée. Cette croissance compensatoire, qui a été observée aussi chez le blé (Lambers et al, 1982), est une réponse de développement, bien connue, à la demande accrue qui pèse sur la partie alimentée du système racinaire. Elle est peut être responsable de l'augmentation de la masse des parties aériennes (fig 3). La présence de $\mathrm{NaCl}$ 
Tableau III. Relation entre la croissance des parties aériennes et l'accumulation de $\mathrm{Na}^{+}$et $\mathrm{Cl}^{-}$. Les teneurs ( $\mu \mathrm{mol} \cdot \mathrm{g}^{-1} \mathrm{MS}$ ) sont mesurées à la récolte finale (24 j). Les masses de matière sèche des parties aériennes (MS) sont tirées des figures 1 et 6 , et exprimées en \% des valeurs des témoins ( $/ T$ ON, et $\mathrm{T} / \mathrm{T} \mathrm{OCa}$ ).

\begin{tabular}{|c|c|}
\hline \multirow{2}{*}{ Traitement } & $\begin{array}{l}\text { Valeurs mesurées dans les parties } \\
\quad \text { aériennes à } t=24 j\end{array}$ \\
\hline & $\begin{array}{ccc}\mathrm{Na}^{+} & \mathrm{Cr} & \mathrm{MS} \\
\left(\mu \mathrm{mol} \cdot \mathrm{g}^{-1} \mathrm{MS}\right) & (\% \text { des témoins })\end{array}$ \\
\hline
\end{tabular}

$\begin{array}{lrrl}\text { T/T ON } & 32 \pm 13 & 291 \pm 70 & 100 \\ \text { T/T12 ON } & 1423 \pm 92 & 1677 \pm 77 & 107 \\ \text { T12/T ON } & 1643 \pm 394 & 2258 \pm 731 & 64 \\ \text { T/T OCa } & 19 \pm 4 & 180 \pm 35 & 100 \\ \text { T/T12 OCa } & 838 \pm 166 & 2181 \pm 670 & 92 \\ \text { T12/T OCa } & 852 \pm 137 & 1735 \pm 443 & 72\end{array}$

autour d'une partie ou de la totalité du système racinaire entraîne une forte diminution de la croissance. Le traitement T12/T oN montre que cette diminution est limitée à $\mathrm{R} 1$ et aux feuilles. La croissance de la partie du système racinaire qui échappe au sel est même stimulée malgré l'absence d'azote dans son milieu (T ON).

\section{Accumulation d'azote}

Les plantes qui peuvent absorber $\mathrm{NO}_{3}^{-}$par un système racinaire sur milieu $T$ accumulent 2,5 à $3 \mathrm{mmol}^{\circ} \mathrm{g}^{-1} \mathrm{MS}$ de $\mathrm{N}$ réduit (tableau IV), et 1,6 à $1,7 \mathrm{mmol} \cdot \mathrm{g}^{-1} \mathrm{MS}$ et $\mathrm{N}$ protéique (tableau $\mathrm{V}$ ) dans les parties aériennes. Par contre, si l'absorption de $\mathrm{NO}_{3}^{-}$ne peut se faire que par des ra-

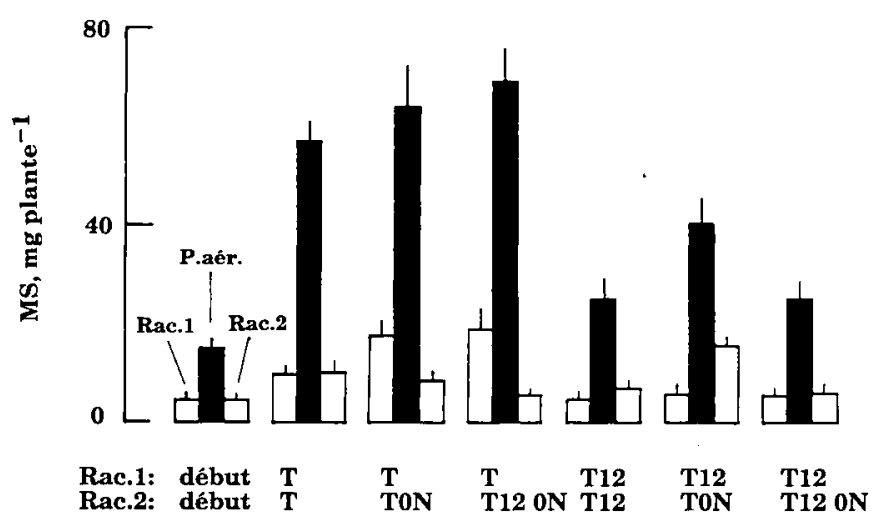

Fig 1. Interactions de $\mathrm{NaCl}$ et $\mathrm{NO}_{3}^{-}$: conséquences sur la croissance. Les histogrammes représentent les masses de matière sèche (MS) des parties aériennes ( $P$ aér) et des deux parties du système racinaire (Rac 1 et Rac 2) placées respectivement sur les milieux 1 et 2 (tableau I). Moyennes de 5 répétitions, intervalles de sécurité au seuil de $95 \%$. cines en présence de $\mathrm{NaCl}$, les niveaux d'accumulation de $\mathrm{N}$ réduit et de $\mathrm{N}$ protéique dans les parties aériennes sont abaissés d'environ $40 \%$. Un phénomène analogue s'observe dans les racines. L'accumulation de $\mathrm{NO}_{3}^{-}$est plus dépendante des limitations de l'absorption de cet ion que ne le sont les accumulations de $\mathrm{N}$ réduit et $\mathrm{N}$ protéique : par exemple, lorsque la plante est obligée d'absorber $\mathrm{NO}_{3}^{-}$en présence de $\mathrm{NaCl}$, la teneur des parties aériennes en $\mathrm{NO}_{3}^{-}$est 10 fois plus faible que dans les traitements où l'absorption est possible à partir du milieu T (tableau IV).

\section{Relation entre la croissance et l'assimilation du nitrate}

La figure 2 met en relation les quantités de matière sèche et d'azote réduit dans les parties aériennes (par plante). Le traitement qui permet l'absorption de $\mathrm{NO}_{3}^{-}$et de $\mathrm{NaCl}$ par des racines différentes ( $T / T 12$ ON) entraîne une aussi bonne croissance que les traitements sans $\mathrm{NaCl}$ ( $\mathrm{T} / \mathrm{T}$ et $T / T$ ON), mais avec une moindre utilisation de $N$ réduit par unité de biomasse produite. La figure 3 est l'analogue de la précédente, mais relative à l'azote protéique au lieu de l'azote réduit. Cette fois tous les traitements sans exception, correspondent à une relation linéaire unique entre les quantités de $\mathrm{N}$ protéique et de matière sèche produite : les traitements ( $\mathrm{ON}$ ou $\mathrm{NaCl}$ ) qui modulent l'alimentation azotée ne touchent pas direc-

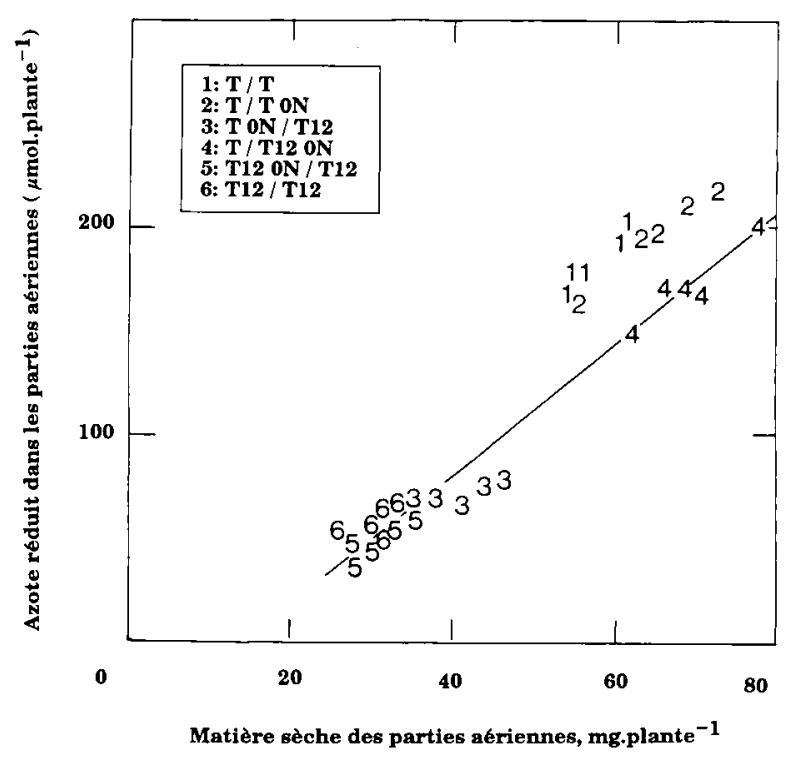

Fig 2. Relation entre la masse de matière sèche et la quantité d'azote assimilé des parties aériennes. Les plantes sont cultivées, avec leurs sytèmes racinaires séparés en deux, sur les traitements indiqués, depuis $12 \mathrm{j}$. A la récolte, elles sont âgées de $24 \mathrm{j}$. Chaque chiffre placé sur la courbe correspond à une plante. 
Tableau IV. Assimilation de $\mathrm{NO}_{3}-$. Les valeurs sont les teneurs en $\mathrm{N}$ réduit et en $\mathrm{NO}_{3}^{-}\left(\mu \mathrm{mol}^{\circ} \mathrm{g}^{-1} \mathrm{MS}\right)$ à $12 \mathrm{j}$ et à $24 \mathrm{j}$. Moyennes de 5 répétitions, intervalles de sécurité au seuil de 95\%. PA : parties aériennes; R1 : racines sur T1; R2 : racines sur T2.

\begin{tabular}{|c|c|c|c|c|c|c|c|}
\hline \multirow{2}{*}{$\begin{array}{c}\text { Traitement } \\
T 1 / T 2 \text { a }\end{array}$} & \multirow{2}{*}{$\begin{array}{c}\text { Temps } \\
\text { (j) }\end{array}$} & \multicolumn{3}{|c|}{$\begin{array}{c}N \text { réd } \\
\left(\mu \mathrm{mol} \cdot \mathrm{g}^{-1} \mathrm{MS}\right)\end{array}$} & \multicolumn{3}{|c|}{$\begin{array}{c}\mathrm{NO}_{3}^{-} \\
\left(\mu \mathrm{mol} \log ^{-1} \mathrm{MS}\right)\end{array}$} \\
\hline & & $P A$ & $R 1$ & $R 2$ & $P A$ & $R 1$ & $R 2$ \\
\hline $\mathrm{T} / \mathrm{T}$ & 12 & $1763 \pm 137$ & $1283 \pm 59$ & $1283 \pm 59$ & 37 & 6 & 6 \\
\hline $\mathrm{T} / \mathrm{T}$ & 24 & $3198 \pm 94$ & $2146 \pm 202$ & $2088 \pm 230$ & 202 & 211 & 195 \\
\hline $\mathrm{T} / \mathrm{T}$ ON & 24 & $3053 \pm 38$ & $2096 \pm 206$ & $1595 \pm 220$ & 228 & 175 & 31 \\
\hline T oN/T12 & 24 & $1755 \pm 146$ & $1128 \pm 106$ & $1449 \pm 99$ & 19 & 4 & 60 \\
\hline $\mathrm{T} / \mathrm{T} 12 \mathrm{ON}$ & 24 & $2505 \pm 124$ & $1893 \pm 83$ & $1069 \pm 96$ & 77 & 123 & 8 \\
\hline $\mathrm{T} 12 / \mathrm{T} 12 \mathrm{ON}$ & 24 & $1621 \pm 134$ & $1318 \pm 78$ & $1245 \pm 36$ & 20 & 38 & 6 \\
\hline $\mathrm{T} 12 / \mathrm{T} 12$ & 24 & $1844 \pm 99$ & $1417 \pm 123$ & $1397 \pm 110$ & 20 & 56 & 54 \\
\hline
\end{tabular}

a La dénomination $\mathrm{T} 1 / \mathrm{T} 2$ indique les milieux sur lesquels croissent respectivement les racines $\mathrm{R} 1$ et $\mathrm{R} 2$.

Tableau V. Accumulation d'azote protéique. Les valeurs sont les teneurs en $\mathbf{N}$ protéique ( $\mu \mathrm{mol} \cdot \mathrm{g}^{-1} \mathrm{MS}$ ) à $12 \mathrm{j}$ et à 24 j. Moyennes de 5 répétitions, intervalles de sécurité au seuil de 95\%. PA : parties aériennes; R1 : racines sur T1; R2 : racines sur $\mathrm{T} 2$.

\begin{tabular}{|c|c|c|c|c|}
\hline \multirow{2}{*}{$\begin{array}{c}\text { Traitement } \\
(T 1 / T 2)^{a}\end{array}$} & \multirow{2}{*}{$\begin{array}{c}\text { Temps } \\
\text { (j) }\end{array}$} & \multicolumn{3}{|c|}{ Teneurs en $N$ protéique $\left(\mu \mathrm{mol} \cdot \mathrm{g}^{-1} \mathrm{MS}\right)$} \\
\hline & & $P A$ & $R 1$ & $R 2$ \\
\hline$T / T$ & 12 & $1014 \pm 63$ & $714 \pm 37$ & $714 \pm 37$ \\
\hline$T / T$ & 24 & $1665 \pm 67$ & $1158 \pm 41$ & $1106 \pm 105$ \\
\hline $\mathrm{T} / \mathrm{T}$ ON & 24 & $1665 \pm 118$ & $976 \pm 106$ & $819 \pm 105$ \\
\hline $\mathrm{T} \mathrm{ON} / \mathrm{T} 12$ & 24 & $1043 \pm 84$ & $668 \pm 67$ & $934 \pm 71$ \\
\hline $\mathrm{T} / \mathrm{T} 12 \mathrm{ON}$ & 24 & $1640 \pm 117$ & $1009 \pm 53$ & $749 \pm 41$ \\
\hline $\mathrm{T} 12 / \mathrm{T} 12 \mathrm{ON}$ & 24 & $925 \pm 181$ & $852 \pm 106$ & $756 \pm 68$ \\
\hline $\mathrm{T} 12 / \mathrm{T}_{12}$ & 24 & $1044 \pm 89$ & $906 \pm 74$ & $922 \pm 87$ \\
\hline
\end{tabular}

a La dénomination T1/T2 indique des milieux sur lesquels croissent respectivement les racines R1 et R2.

tement la nutrition protéique. Le fait que les points du traitement $\mathrm{T} / \mathrm{T} 12$ oN s'écartent de l'alignement des autres sur la figure 2 ( $N$ réduit = $\mathrm{f}(\mathrm{MS})$ mais non sur la figure 3 ( $N$ protéique = $f(M S)$ ) indique que lorsque $\mathrm{NaCl}$ et $\mathrm{NO}_{3}^{-}$sont fournis séparément, une production donnée de matière sèche nécessite autant de $\mathrm{N}$ protéique, mais moins d'azote réduit que dans les autres traitements. Ceci montre que $\mathrm{NaCl}$, lorsqu'il ne gêne pas l'approvisionnement en $\mathrm{NO}_{3}^{-}$permet à la plante d'économiser de l'azote réduit soluble (différence entre $\mathrm{N}$ réduit et $\mathrm{N}$ protéique), en augmentant le rendement de son utilisation pour la croissance.

\section{Conclusion}

Le chlorure de sodium agit de deux façons sur la nutrition azotée de l'orge :

- il limite l'approvisonnement en $\mathrm{NO}_{3}^{-}$, par effet direct sur la racine. Ceci abaisse la teneur des tissus en composés azotés et limite éventuellement leur croissance;

- il est capable d'améliorer le rendement d'utilisation de l'azote soluble réduit pour la croissance, conduisant ainsi à une économie qui limite la baisse de croissance évoquée ci-dessus. II est possible que $\mathrm{Na}^{+}$et $\mathrm{Cl}^{-}$remplacent des 


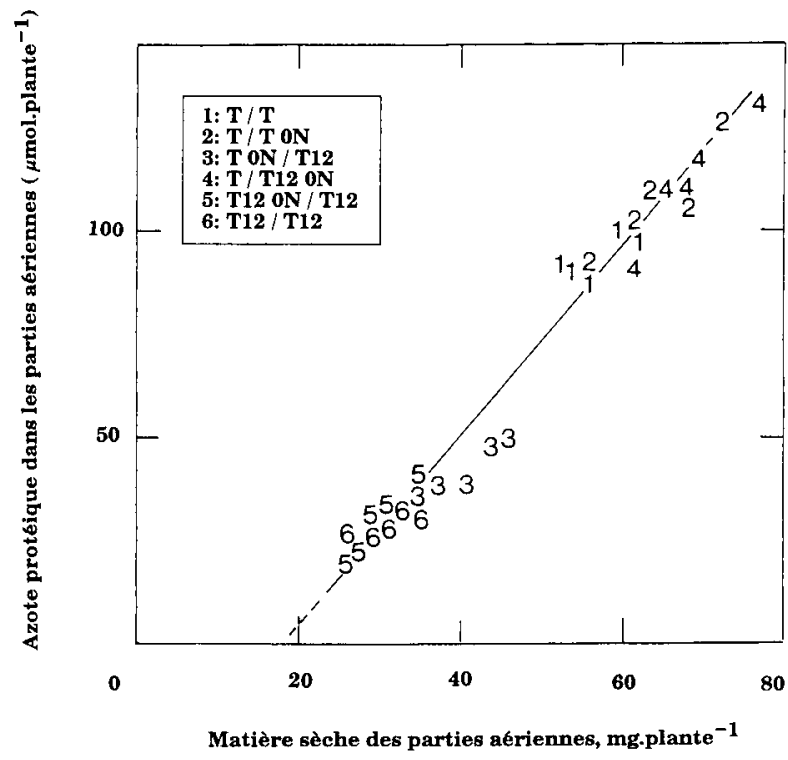

Fig 3. Relation entre la masse de matière sèche $\left(\mathrm{mg}^{\cdot}\right.$ plante $\left.^{-1}\right)$ et la quantité d'azote protéique ( $\mu \mathrm{mol}^{-p} \mathrm{plante}^{-1}$ ) des parties aériennes. Les plantes sont cultivées avec leurs systèmes racinaires séparés en deux, sur les traitements indiqués, depuis $12 \mathrm{j}$. A la récolte elles sont âgées de $24 \mathrm{j}$. Chaque chiffre sur la courbe correspond à une plante.

composés azotés utilisés comme osmoticums et les libèrent pour la synthèse protéique.

L'illustration la plus claire de ces effets est obtenue avec le traitement $\mathrm{T} / \mathrm{T} 12$ oN qui autorise l'effet bénéfique de $\mathrm{NaCl}$ et évite ses effets inhibiteurs (fourniture séparée de $\mathrm{NO}_{3}^{-}$et de $\mathrm{NaCl}$ ). Bien que bloquant la croissance de la racine sur $\mathrm{NaCl}$, ce traitement entraîne une production totale de matière sèche équivalente à celles des traitements témoins sans $\mathrm{NaCl}$ (fig 1) et maintient une bonne alimentation azotée (fig 2). Le traitement $\mathrm{T} 0 \mathrm{~N} / \mathrm{T} 12$ qui ne diffère de $\mathrm{T} / \mathrm{T} 12 \mathrm{ON}$ que par le fait que $\mathrm{NO}_{3}^{-}$doit être absorbé en présence de $\mathrm{NaCl}$, entraîne au contraire une forte réduction de la teneur en azote protéique (tableau V, fig 3) et de la croissance. Étant donné que l'alimentation en $\mathrm{K}$ et autres éléments essentiels reste assurée par le milieu $T \mathrm{ON}$, ce résultat indique que l'effet inhibiteur de $\mathrm{NaCl}$ sur l'absorption de $\mathrm{NO}_{3}^{-}$est suffisant pour limiter la croissance des plantes.

\section{Alimentation en potassium}

L'omission de $\mathrm{K}+$ sur une moitié du système racinaire alors que l'autre est soit sur $T$, soit sur $T 12$, a un faible effet sur la croissance des feuilles (fig 4) : les diminutions par rapport au traitement $T / T$ sont respectivement de $7 \%$ et $9 \%$ pour les traite-
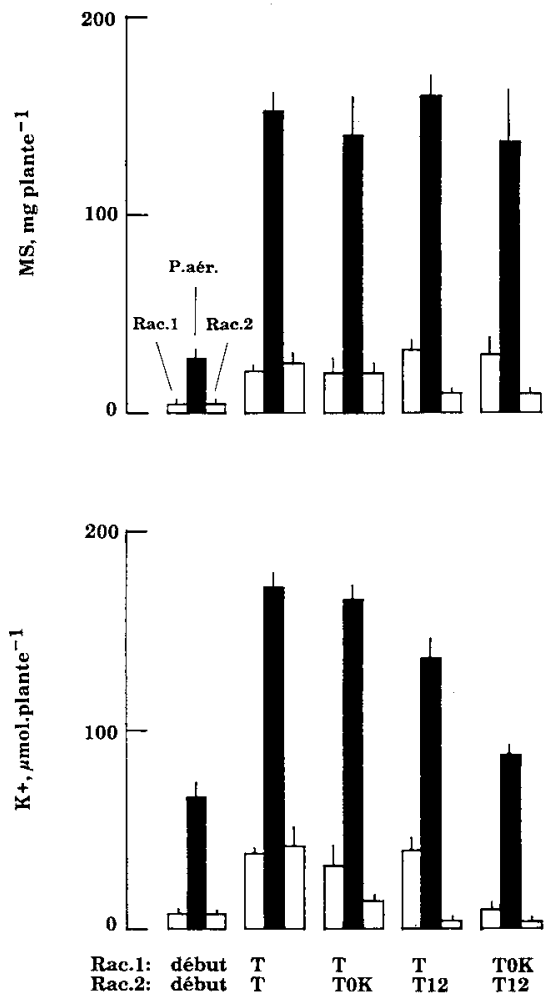

Fig 4. Effet de la déficience en $\mathrm{K}$. En haut : Masse de matière sèche (mg-plante ${ }^{-1}$ ) des parties aériennes et de chacune de deux moitiés du système racinaire à l'âge de 12 j (début) et de $24 \mathrm{j}$. En bas : quantités ( $\mu$ mol-plante ${ }^{-1}$ ) de $\mathrm{K}^{+}$ correspondantes. Moyennes de 5 répétitions. Les barres verticales correspondent aux intervalles de sécurité au seuil de $95 \%$. Pour le traitement de chaque moitié racinaire, of tableau II.

ments $T / T$ OK et $T 12 / T$ OK; les parties de système racinaire privées de $\mathrm{K}+$ ont une croissance normale ou légèrement stimulée (fig 4). En outre, la comparaison des traitements où une partie du système racinaire peut absorber librement $\mathrm{K}+(\mathrm{T} /$ $T$ OK et $T / T 12)$, et du traitement qui en limite l'absorption des deux côtés du système racinaire (T12/T OK), montre que la croissance des feuilles n'est pas affectée par ce dernier traitement, malgré une forte diminution des teneurs en $\mathrm{K}^{+}$(tableau $\mathrm{Vl}$ ). Ceci suggère que la réduction de l'absorption de $\mathrm{K}^{+}$n'est pas le facteur limitant de la croissance en présence $\mathrm{NaCl}$ dans le milieu (fig 4).

L'effet de la déficience en potassium induite par le sel a été apprécié par des expériences dans lesquelles on a eu recours à un apport supplémentaire de $\mathrm{K}^{+}(8 \mathrm{mM})$ au milieu de culture contenant $\mathrm{NaCl}, 12 \mathrm{~g}^{\circ} \mathrm{l}^{-1}(200 \mathrm{mM})$. La figure 5 montre que l'addition de $\mathrm{K}+8 \mathrm{mM}$ au milieu salé $(T 12+K)$ n'améliore pas la croissance des plantes bien que l'absorption de $\mathrm{K}+$ soit augmentée de manière appréciable. II s'ensuit donc un 
Tableau VI. Accumulation de $\mathrm{K}^{+}$. Les teneurs en $\mathrm{K}^{+}$ ( $\mu \mathrm{mol} \cdot \mathrm{g}^{-1} \mathrm{MS}$ ) des parties aériennes et des deux moitiés (1) et (2) du système racinaire sont mesurées sur des plantes âgées de 24 j. Moyennes de 5 répétitions; intervalles de sécurité au seuil de $95 \%$.

\begin{tabular}{|c|c|c|c|}
\hline \multirow{2}{*}{$\begin{array}{c}\text { Traitement } \\
T 1 / T 2^{a}\end{array}$} & \multicolumn{3}{|c|}{ Teneurs en $\mathrm{K}^{+}\left(\mu \mathrm{mol} \cdot \mathrm{g}^{-1} \mathrm{MS}\right)$} \\
\hline & $P A$ & Racine 1 & Racine 2 \\
\hline $\mathrm{T} / \mathrm{T}$ & $1125 \pm 71$ & $1746 \pm 138$ & $1646 \pm 81$ \\
\hline $\mathrm{T} / \mathrm{T} \mathrm{OK}$ & $1179 \pm 114$ & $1603 \pm 125$ & $714 \pm 150$ \\
\hline $\mathrm{T} / \mathrm{T} 12$ & $853 \pm 93$ & $1254 \pm 167$ & $408 \pm 65$ \\
\hline TOKT12 & $648 \pm 101$ & $328 \pm 55$ & $362 \pm 101$ \\
\hline
\end{tabular}

a La dénomination $T 1 / T 2$ indique les milieux sur lesquels croissent les racines $R 1$ et $R 2$.
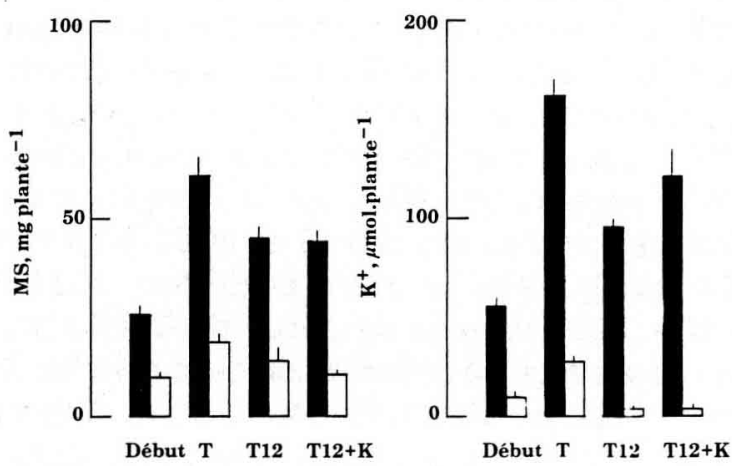

Fig 5. Masse de matière sèche et quantités de K accumulées dans les parties aériennes et les racines. Début : prélèvement initial (plantes âgées de $12 \mathrm{j}$, sur milieu témoin sans sel). T, T12 et T12 + K : plantes âgées de $24 \mathrm{j}$, cultivées respectivement sur milieu additionné de $\mathrm{NaCl}(200 \mathrm{mM})$, et sur milieu avec $\mathrm{NaCl}$ et enrichi en $\mathrm{K}+(8 \mathrm{mM})$. Moyennes de 5 répétitions, intervalles de sécurité au seuil de $95 \%$. Noir : parties aériennes; blanc : racines.

enrichissement des tissus en $\mathrm{K}+$. L'augmentation de l'absorption de $\mathrm{K}+$ indique que $\mathrm{NaCl}$ a rendu la concentration de $\mathrm{K}+$ du milieu limitante par rapport aux capacités d'absorption de la plante, mais les sytèmes d'absorption, même limités, fournissent à la plante une quantité de $\mathrm{K}^{+}$suffisante à la croissance.

\section{Alimentation en calcium}

La suppression de $\mathrm{Ca}^{2+}$ du milieu de culture, sur une partie du système racinaire, a été faite selon la même procédure que pour $\mathrm{K}^{+}$(tableau II). La figure 6 permet de distinguer deux catégories de traitements : ceux qui autorisent l'absorption de

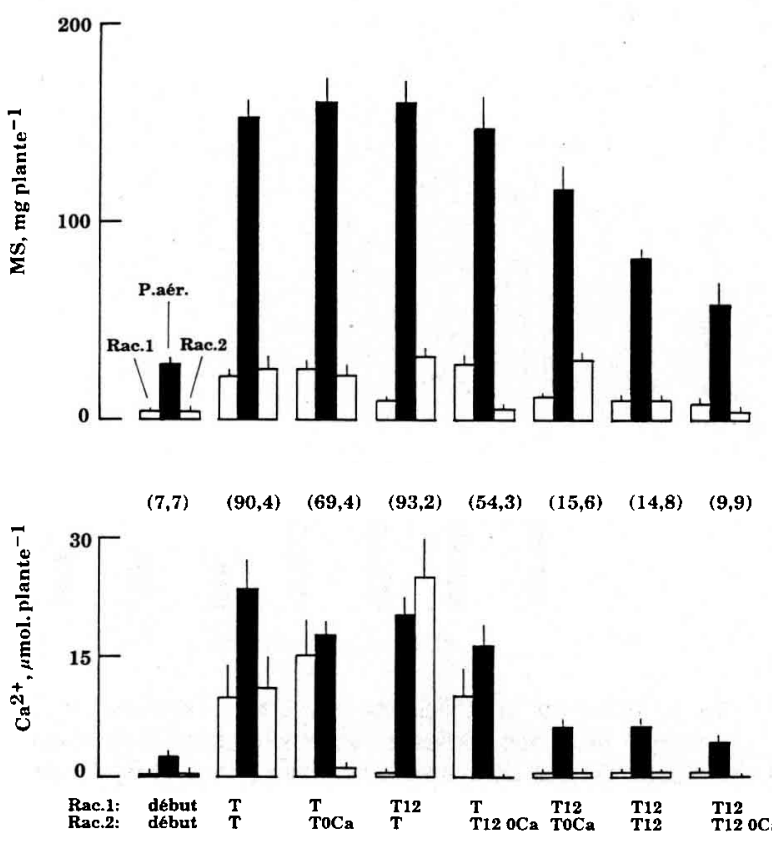

Fig 6. Effet de la déficience en $\mathrm{Ca}^{2+}$. En haut : Masse de matière sèche $\left(\mathrm{mg} \cdot\right.$ plante $\left.^{-1}\right)$ des parties aériennes ( $P$ aér) et de chacune des deux moitiés 1 et 2 du système racinaire (Rac1 et Rac2) à l'âge de $12 \mathrm{j}$ (début) et de $24 \mathrm{j}$. En bas : quantités correspondantes ( $\mu$ eq plante $^{-1}$ ) de $\mathrm{Ca}^{2+}$. Moyennes de 5 répétitions, les barres verticales correspondent aux intervalles de sécurité au seuil de $95 \%$. Pour le traitement de chaque moitié racinaire, cf tableau II.

$\mathrm{Ca}^{2+}$ en l'absence de $\mathrm{NaCl}(\mathrm{T} / \mathrm{T} ; \mathrm{T} / \mathrm{T}$ OCa; $\mathrm{T} 12 /$ $\mathrm{T} ; \mathrm{T} / \mathrm{T} 12 \mathrm{OCa}$ ) et ceux qui obligent la plante à le prélever en présence de $\mathrm{NaCl}$ (T12/T12; T12/T $0 \mathrm{Ca} ; \mathrm{T} 12 / \mathrm{T} 12 \mathrm{OCa})$. Les premiers donnent une croissance plus forte que les seconds. En milieu témoin, la suppression de $\mathrm{Ca}^{2+}$ sur une partie du système racinaire $(\mathrm{T} / \mathrm{T} \mathrm{OCa})$ n'a pas d'effet sur la croissance des différents organes et même de la partie du système racinaire privée de $\mathrm{Ca}^{2+}$. Bien que la teneur en $\mathrm{Ca}^{2+}$ des feuilles, et des racines sur $\mathrm{T} \mathrm{OCa}$, soit abaissée par rapport au témoin (fig 7), la partie du système racinaire sur milieu $T$ assure à elle seule une alimentation de la plante en $\mathrm{Ca}^{2+}$ convenable pour la croissance.

L'addition de $\mathrm{NaCl}$ au milieu n'inhibe pas la croissance des parties aériennes si une partie du système racinaire reste sur $T$ (comparer $T / T$ et $T / T 12$ avec T12/T12). Néanmoins, les racines au contact de $\mathrm{NaCl}$ présentent une forte réduction de croissance et de teneur en $\mathrm{Ca}^{2+}$ (divers traitements T12, figs 6 et 7). It est clair que l'un des effets du sel est de diminuer l'absorption de $\mathrm{Ca}^{2+}$. Lorsque cet effet s'exerce sur tout le système racinaire, ou bien lorsqu'il est complété par l'absence de $\mathrm{Ca}^{2+}$ dans le milieu, les quantités 


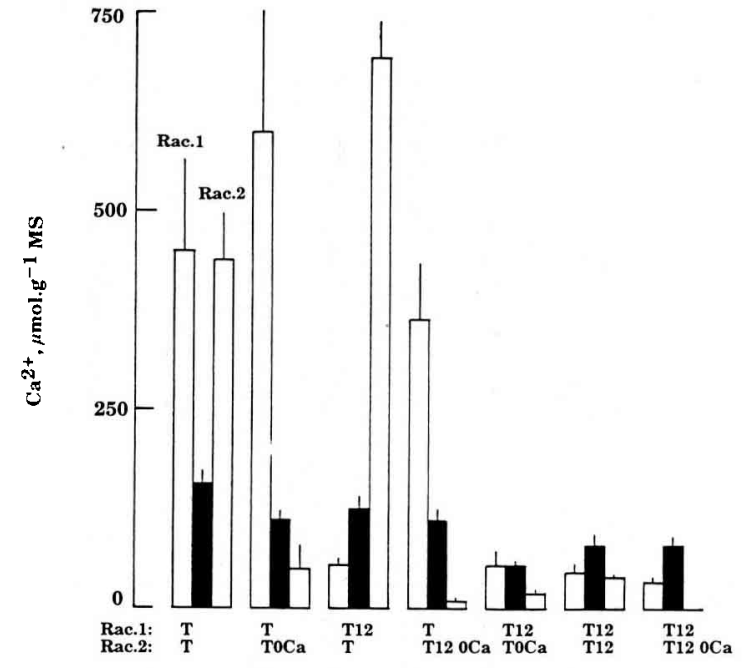

Fig 7. Effet de la déficience en $\mathrm{Ca}^{2+}$. Teneurs en $\mathrm{Ca}^{2+}$ ( $\mu \mathrm{mol} \cdot \mathrm{g}^{-1} \mathrm{MS}$ ) des parties aériennes (en noir) el de chacune des deux moitiés du système racinaire (Rac1 et Rac2) mesurées à l'âge de $24 \mathrm{j}$. Moyennes de 5 répétitions, les barres verticales correspondent aux intervalles de sécurité au seuil de $95 \%$. Les deux moitiés du système racinaire proviennent respectivement des milieux 1 et 2 (cf tableau II).

totales de $\mathrm{Ca}^{2+}$ dans les parties aériennes sont très fortement abaissées de même que la croissance (fig 6). Par exemple, le traitement T12/T $0 \mathrm{Ca}$, qui ne diffère du traitement $\mathrm{T} / \mathrm{T} 12 \mathrm{OCa}$ que par le fait que $\mathrm{Ca}^{2+}$ doit être absorbé obligatoirement en présence de $\mathrm{NaCl}$ entraîne une réduction de la croissance de $15 \%$ par rapport à lui, et supprime pratiquement l'absorption de $\mathrm{Ca}^{2}+$ pendant la durée du traitement (comparer les quantités de $\mathrm{Ca}^{2}+\mathrm{a} t=12 \mathrm{j}$ et $\mathrm{t}=24 \mathrm{j}$ ). Ceci indique que l'effet inhibiteur de $\mathrm{NaCl}$ sur l'absorption de $\mathrm{Ca}^{2+}$ est suffisant à lui seul pour limiter la croissance des plantes, étant donné que l'alimentation en $\mathrm{K}^{+}, \mathrm{NO}_{3}^{-}$et autres ions essentiels reste assurée (milieu $\mathrm{T} \mathrm{OCa}$, fig 6). En conclusion, $\mathrm{NaCl}$ affecte la croissance en limitant l'approvisionnement de la plante en calcium.

\section{DISCUSSION ET CONCLUSION}

Dans les expériences présentées ici les différents traitements sont appliqués pendant 12 jours. Les résultats montrent que si $\mathrm{NaCl}(200$ $\mathrm{mM}$ ) inhibe le prélèvement de $\mathrm{N}, \mathrm{Ca}$ et $\mathrm{K}$, seuls les deux premiers éléments sont devenus limitants pour la croissance. Cette inhibition ne se fait que si le sel est présent dans le milieu où baigne la racine chargée d'alimenter la plante. $\mathrm{Si}$ l'absorption de l'élément étudié peut se faire par une partie du système racinaire dans un milieu sans $\mathrm{NaCl}$, l'alimentation est normale en dépit d'une forte absorption de $\mathrm{NaCl}$ par le reste des racines. Donc c'est $\mathrm{NaCl}$ du milieu et non celui accumulé dans les tissus qui est responsable de l'inhibition de l'absorption.

Chez l'orge, le potassium est très abondant par rapport aux cations bivalents, $\mathrm{Ca}^{2+}, \mathrm{Mg}^{2+}$ (Coïc et al, 1962; Greenway 1962, 1963 et 1965) probablement à cause de la faible capacité d'échange cationique racinaire de cette plante (Drake et al, 1951). Cependant, en condition de stress salin, le prélèvement de $\mathrm{K}^{+}$, même faible, suffit à assurer les besoins de la croissance, malgré l'enrichissement des tissus en $\mathrm{Na}^{+}$qui devient alors le cation prépondérant. Avec $\mathrm{K}^{+}$et $\mathrm{Cl}^{-}$, il contribue pour 50 à $60 \%$ à l'ajustement osmotique (Wyn Jones et Story, 1978; Delane et al, 1982). L'enrichissement des tissus en $\mathrm{Na}^{+}$serait plutôt la conséquence que la cause de là réduction de la croissance (Delane et al, 1982). Les résultats présentés ici, montrent par contre, que $\mathrm{NaCl}$ exerce son effet dépressif sur la croissance en limitant l'approvisionnement de la plante en $\mathrm{Ca}^{2+}$. L'adjonction de sels de calcium diminue l'effet dépressif de $\mathrm{NaCl}$ sur la croissance des plantes en milieu très chargé en $\mathrm{NaCl}$ (Heyder et Greenway, 1965; La Haye et Epstein, 1969 et 1971). Mais cet effet bénéfique de $\mathrm{Ca}^{2+}$ a souvant été attribué à la diminution de la perte de $\mathrm{K}^{+}$ (provoquée par $\mathrm{NaCl}$ ) du tissu racinaire (Cramer et al, 1985; Kent et Läuchli, 1985; Hajji et Grignon, 1985). Les résultats présentés ici montrent que l'inhibition de l'alimentation calcique par $\mathrm{NaCl}$, peut avoir un effet direct sur la croissance, indépendamment de l'alimentation potassique, qui reste assurée par le système d'alimentation mixte.

Enfin, en ce qui concerne $\mathrm{N}$, on sait que l'augmentation de la concentration du milieu en $\mathrm{NO}_{3}^{-}$ peut améliorer la croissance des plantes traitées par $\mathrm{NaCl}$ (Langdale et Thomas, 1971). Cet effet a été attribué à une réduction de l'absorption de $\mathrm{Cl}$ par $\mathrm{NO}_{3}^{-}$, plutôt qu'à la levée de la limitation de la fourniture d'azote (Torres et Bingham, 1973; Kafkafi et al, 1982; Youssef et Sprint, 1983; Glass et Siddiqi, 1985; Munns et Termaat, 1986). Mais nos résultats, qui permettent de comparer les comportements de plantes absorbant $\mathrm{NO}_{3}^{-}$et $\mathrm{NaCl}$ par la même racine ou par des racines distinctes, confirment que le sel peut limiter la croissance en restreignant la disponibilité de $\mathrm{NO}_{3}^{-}$.

En résumé, cette étude montre que $\mathrm{NaCl}$, appliqué à la concentration qui correspond au seuil de tolérance de l'orge, limite l'approvisionnement 
de la plante en éléments essentiels comme K, $\mathrm{Ca}$ et $\mathrm{N}$. Il est évidemment possible que d'autres éléments soient impliqués dans cet effet du sel. L'approche utilisée permet de distinguer les effets propres de chacun des trois éléments étudiés. En absence de $\mathrm{NaCl}$, la composition du milieu $T$ assure qu'aucun de ces éléments n'est limitant pour la croissance. En présence de $\mathrm{NaCl}$, les accumulations de $\mathrm{Na}^{+}$et $\mathrm{Cl}-$ dans les tissus ne sont pas impliquées dans la limitation de l'alimentation et de la croissance des plantes. C'est par sa présence dans le milieu, et par son effet direct sur la racine que $\mathrm{NaCl}$ restreint l'approvisionnement des plantes en $\mathrm{NO}_{3}^{-}, \mathrm{Ca}^{2+}$ et $\mathrm{K}^{+}$ jusqu'à un niveau limitant la croissance pour les deux premiers nutriments, mais non pour le troisième. Évidemment, on peut s'attendre à ce que ces résultats dépendent, pour chaque nutriment, de son abondance dans le milieu, et du besoin de la plante (c'est-à-dire, de la vitesse de prélèvement minimum, nécessaire pour soutenir la croissance normale). Dans le cas présent, on peut estimer d'après les teneurs (tableaux IV et $\mathrm{VI}$, fig 7), que le besoin en $\mathrm{K}^{+}$est du même ordre de grandeur que le besoin en $\mathrm{N}$, et nettement supérieur au besoin en $\mathrm{Ca}^{2+}$. En dépit de cela, et bien qu'il était presque deux fois moins représenté dans les milieux que $\mathrm{NO}_{3}^{-}$et $\mathrm{Ca}^{2+}, \mathrm{K}^{+}$n'a pas été un nutriment limitant pour la croissance en présence de $\mathrm{NaCl}$.

\section{RÉFÉRENCES}

Aslam M, Huffaker RC, Rains DW (1984) Early effects of salinity on nitrate assimilation in barley seedlings. Plant Physiol 76, 321-325

Coïc Y, Lesaint C, Piollat MT (1962) Influence de la déficience en potassium et de la déficience en eau sur la composition minérale de l'orge. Ann Physiol Vég 4, 227-234

Cramer GR, Laüchli A, Polito VS (1985) Displacement of $\mathrm{Ca}^{2+}$ by $\mathrm{Na}^{+}$from the plasmalemma of root cells. A primary response to salt stress ? Plant Physiol 79, 207-211

Cramer GR, Läuchli A, Epstein E (1986) Effects of $\mathrm{NaCl}$ and $\mathrm{CaCl}_{2}$ on ion activities in complex nutrients solutions and root growth of cotton. Plant Physio/ 81, 792-797

Deane-Drummond DE, Glass ADM (1982) Studies of nitrate influx into barley roots by the use of ${ }^{36} \mathrm{ClO}_{3}-$ as a tracer for nitrate. I. Interactions with chloride and other ions. Can J Bot 60, 2147-2153

Delane R, Greenway H, Munns R, Gibbs J (1982) Ion concentration and carbohydrate status of the elongating leaf tissue of Hordeum vulgare growing at high external $\mathrm{NaCl}$. I. Relationship between solute concentration and growth. J Exp Bot 33, 557-573
Drake M, Vengris J, Colby WG (1951) Cationexchange capacity of plant roots. Soil Sci 72, 139147

Elzam OE (1971) Interaction between sodium, potassium and calcium in their absorption by intact barley plants. In: Recent Advances in Plant Nutrition (Samish RM, ed) Gordon and Breach Science Publ Inc, NY 2, 491-507

Glass ADM, Siddiqi MY (1985) Nitrate inhibition of chloride influx in barley: implication for a proposed chloride homeostat. J Exp Bot 36, 556-566

Greenway H (1962a) Plant response to saline substrates. I. Growth and ion uptake of several varieties of Hordeum during and after sodium chloride treatment. Aust J Biol Sci 15, 16-38

Greenway H (1962b) Plant responses to saline substrates. II. Chloride, sodium and potassium uptake and translocation in young plants of Hordeum vulgare during and after short sodium chloride treatment. Aust J Biol Sci 15, 39-57

Greenway H (1963) Plant responses to saline substrates. III. Effect of nutrient concentration on the growth and ion uptake of Hordeum vulgare during a sodium chloride stress. Aust J Biol Sci 16, 616628

Greenway H (1965) Plant responses to saline substrates. VII. Growth and ion uptake throughout plant development in two varieties of Hordeum vulgare. Aust J Biol Sci 18, 763-779

Hajji M (1980) La responsabilité de la racine dans la sensibilité du laurier-rose au chlorure de sodium. Physiol Vég 18, 505-515

Hajji M, Grignon C (1985) Identification des transports de $\mathrm{K}^{+}\left(\mathrm{Rb}^{+}\right)$affectés par $\mathrm{NaCl}$ dans la racine du laurier-rose. Physiol Vég 23, 3-12

Helal HM, Mengel K (1979) Nitrogen metabolism of young barley plants as affected by $\mathrm{NaCl}$-salinity and potassium. Plant Soil 51, 457-467

Heyder SZ, Greenway H (1965) Effect of $\mathrm{Ca}^{2+}$ on plant sensitivity to high $\mathrm{NaCl}$ concentrations. Plant Soil 23, 258-260

Kafkafi U, Valoras N, Letey J (1982) Chloride interaction with nitrate and phosphate nutrition in tomato (Lycopersicon esculentum L). J Plant Nutr 5, 13691385

Kent L.M, Läuchi A (1985) Germination and seedling growth of cotton: salinity-calcium interactions. Plant Cell Environ 8, 155-159

La Haye PA, Epstein E (1969) Salt toleration by plants: enhancement with calcium. Science 166, 395-396

La Haye PA, Epstein E (1971) Calcium and salt toleration by bean plants. Physiol Plant 25, 213-218

Lambers H, Simpson RJ, Beilhartz VC, Dalling MJ (1982) Growth and translocation of $C$ and $N$ in wheat (Triticum aestivum) grown with a split root system. Physiol Plant 56, 421-429

Langdale GW, Thomas JR (1971) Soil salinity effects in absorption of nitrogen, phosphorus and protein synthesis by coastal Bermuda grass. Agron $J 63$, 708-711 
Lucque AA, Bingham FT (1981) The effect of the osmotic potential and specific ion concentration on the nutrient solution on the uptake and reduction of nitrate by barley seedlings. Plant Soil 63, 227-237

Lynch J, Läuchli A (1986) Salinity affects intracellular calcium in corn root protoplast. Plant Physiol 87, 351-356

Munns R, Greenway H, Delane R, Gibbs J (1982) Ion concentration and carbohydrate status of the elongating leaf-tissue of Hordeum vulgare growing at high external $\mathrm{NaCl}$. Il. Cause of the growth reduction. J Exp Bot 33, 574-583

Munns R, Termaat A (1986) Whole-plant responses to salinity. Aust J Plant Physiol 13, 143-160

Soll H, Bottger M (1982) The mechanism of protoninduced increase of cell wall extensibility. Plant Sci Lett 24, 163-171

Soltani A, Hajij M, Grignon C (1989) Nécessité d'un anion exogène en cas de nutrition ammoniacale. Agronomie 9, 777-784
Stassart JM, Neirinckx L, Dejaegere R (1981) The interactions between monovalent cations and calcium during their adsorption on isolated cell walls and absorption by intact barley roots. Ann Bot 47, 647-652

Torres BC, Bingham FT (1973) Salt tolerance of Mexican wheat: I. Effect of $\mathrm{NO}_{3}$ and $\mathrm{NaCl}$ on mineral nutrition, growth and grain production of four wheats. Soil Sci Soc Am Proc 37, 711-715

Wyn Jones RG, Storey R (1978) Stress and comparative physiology in the Graminaea. IV. Comparison of salt stress in Spartina townsendii and three barley cultivars. Aust J Plant Physiol 5, 839-850

Youssef AN, Sprint Jl (1983) Effect of $\mathrm{NaCl}$ on growth, nitrogen incorporation and chemical composition of inoculated and $\mathrm{NH}_{4} \mathrm{NO}_{3}$ fertilised Vicia faba $\mathrm{L}$ plants. J Exp Bot 34, 941-650

Zid E, Grignon C (1985) Sodium-calcium interactions in leaves of Citrus aurantium grown in the presence of NaCl. Phys Vég 23, 895-903 